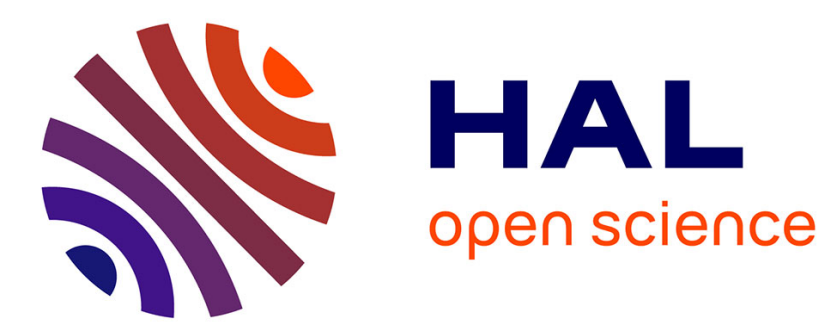

\title{
Theoretical calculation of Tc for lead
}

\author{
A.D. Zdetsis, E.N. Economou, D.A. Papaconstantopoulos
}

\section{To cite this version:}

A.D. Zdetsis, E.N. Economou, D.A. Papaconstantopoulos. Theoretical calculation of Tc for lead. Journal de Physique Lettres, 1979, 40 (12), pp.253-255. 10.1051/jphyslet:019790040012025300 jpa00231619

\section{HAL Id: jpa-00231619 https://hal.science/jpa-00231619}

Submitted on 1 Jan 1979

HAL is a multi-disciplinary open access archive for the deposit and dissemination of scientific research documents, whether they are published or not. The documents may come from teaching and research institutions in France or abroad, or from public or private research centers.
L'archive ouverte pluridisciplinaire HAL, est destinée au dépôt et à la diffusion de documents scientifiques de niveau recherche, publiés ou non, émanant des établissements d'enseignement et de recherche français ou étrangers, des laboratoires publics ou privés. 


\title{
Theoretical calculation of $T_{c}$ for lead $\left({ }^{*}\right)\left({ }^{* *}\right)$
}

\author{
A. D. Zdetsis, E. N. Economou (***) \\ N.R.C. Demokritos, Aghia Paraskevi, Attikis, Greece \\ and D. A. Papaconstantopoulos \\ Naval Research Laboratory, Washington, D.C., U.S.A. \\ (Reçu le 10 août 1978, révisé le 2 avril 1979, accepté le 24 avril 1979)
}

\begin{abstract}
Résumé. - Nous calculons la température de transition supraconductrice du $\mathrm{Pb}$ avec la théorie de Gaspari et Gyorffy, en utilisant nos calculs ab initio de structure de bande. La valeur calculée ainsi est la moitié de la valeur expérimentale. Cet écart est attribué à l'approximation du potentiel muffin tin rigide. Pour tenir compte de cette approximation nous présentons une méthode simple, sans paramètre ajustable. La nouvelle valeur calculée de la température de transition est en accord excellent avec la valeur expérimentale.
\end{abstract}

\begin{abstract}
Using our ab initio band structure results, we calculate the transition temperature of $\mathrm{Pb}$ according to the Gaspari-Gyorffy theory. The calculated value is half the experimental one. The discrepancy is attributed to the rigid muffin tin approximation. A simple method with no adjustable parameters is developed to account for this approximation. The new transition temperature is in excellent agreement with experiment.
\end{abstract}

Following the work of McMillan [1], which relates in a practical way the superconducting transition temperature $T_{\mathrm{c}}$ to microscopic quantities, a large amount of research has been reported along those lines. In particular, the theory of Gaspari and Gyorffy [2, 3], which we employ here, was designed especially for transition metals. However, as we shall illustrate below, this theory properly corrected can give very good estimates of the electron-phonon interaction $\eta$ for normal metals like lead as well. The parameter $\eta$, which together with the average phonon frequency $\left\langle\omega^{2}\right\rangle$, [1, 4] defines $T_{\text {c }}$, can be expressed in the Gaspari-Gyorffy theory in terms of partial densities of states and phase shifts readily available from traditional band structure calculations.

In the first stage of this work we have determined, within the Rigid Muffin Tin Approximation (RMTA), the quantity $\eta$ using our self-consistent $a b$ initio band calculation. The latter includes relativistic effects, such as the mass velocity and Darwin corrections,

(*) Partly supported by NSF grant No. DMR 76-19458 at the University of Virginia.

$\left.{ }^{(* *}\right)$ This paper was presented at the LT 15 Conference as a post-deadline paper.

$\left({ }^{* * *}\right)$ On leave from the Dept. of Physics, University of Virginia, Charlottesville, Virginia 22901, U.S.A. which for a heavy metal such as $\mathrm{Pb}$ are important $[5,6]$. The spin orbit interaction has been neglected because, although it is very important for the structure of the Fermi surface, is not expected to be very significant for the electron-phonon interaction, and consequently for $T_{c}$, which is our primary concern here. Details of our band structure calculation, together with comparisons with previous calculations and experimental data are published elsewhere [7].

The total and partial densities of states $n_{\mathrm{t}}$ and $n_{l}$

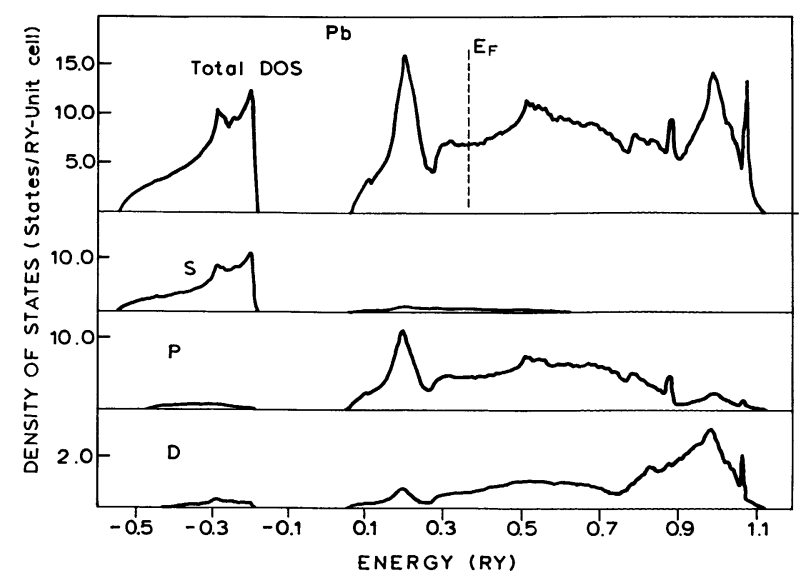

Fig. 1. - Electronic density of states (DOS) of $\mathrm{Pb}$ and its s-, pand d-like components. 
respectively, which are calculated by our modified QUAD method [8], are shown in figure 1. The same quantities evaluated at the Fermi level $E_{\mathrm{F}}$, together with the scattering phase shift $\delta_{l}$, the mass enhancement $\lambda$ and the free scatterer partial densities of states $n_{l}^{(1)}$ are listed in table I. We have calculated $T_{\mathrm{c}}$ according to the Allen and Dynes equation [4] with $M\left\langle\omega^{2}\right\rangle$ the phononic contribution to $\lambda$, taken from their paper. The Coulomb potential $\mu^{*}$ was evaluated by the Bennemann and Garland formula [9]. The experimental value of $\lambda$, measured through tunnelling experiments (see refs. $[4,10]$ ) is twice as large as our ab initio value calculated here.

This discrepancy must be due to the inherent approximations of the Gaspari-Gyorffy (GG) theory; namely the spherical, the local and the rigid muffin tin approximation $[2,3,11]$. The first two approximations are not expected to be important for lead. The spherical approximation is known to be well satisfied for cubic crystals and $l=0,1,2$ [12].

The local approximation for lead is also expected to be well obeyed due to the large contribution of Umklapp electron-phonon scattering [11, 13-15]. It has been demonstrated [11-13, 14] that the local approximation is better satisfied for large Umklapp scattering. Furthermore, the validity of the local approximation can be also verified from the similarity of the phonon density of states curve, $F(\omega)$ and the $\alpha^{2}(\omega) F(\omega)$ profile [11].

The Eliashberg function $\alpha^{2}(\omega) F(\omega)$ defined in ref. [1] has been determined experimentally by superconducting tunnelling measurements (see refs. $[4,10]$ ). Thus, the discrepancy between the observed and calculated values of $\lambda$ and $T_{\mathrm{c}}$ must be mainly attributed to the rigid muffin tin approximation (RMTA). The validity of RMTA depends on the importance of the tails of the self consistent potential about the edge of the muffin-tin sphere. For transition metals, where the scattering of electrons is dominated by the atomic potential inside the muffin tin sphere, this approximation is expected to be quite good.
For lead, however, one must go beyond this approximation by adding a correction term to the RMTA potential. On physical grounds this correction term $V_{\mathrm{c}}(r)$ is expected to have a strong influence near the muffin-tin radius, $r_{\mathrm{MT}}$, and decrease rapidly away from the muffin-tin sphere. Therefore a plausible choice for $V_{\mathrm{c}}(r)$ is

$$
V_{\mathrm{c}}(r)=\frac{V_{0}}{\mathrm{e}^{q_{\mathrm{TF}}\left(r-r_{\mathrm{MT}}\right)}+1}
$$

where $q_{\mathrm{TF}}$ is the inverse of the Thomas-Fermi screening length and $V_{0}$ is an as yet unspecified parameter which denotes the depth of the correction potential.

To determine $V_{0}$ without any resort to experimental information, we have adapted the equivalent RMTA electron-phonon pseudopotential of Lee and Heine [16] which has been expressed in terms of the same quantities that appear in the GG formula [11]. This pseudopotential in $q$-space has the form [11]

$$
\begin{aligned}
\tilde{V}_{\mathrm{RMTA}}(q)=\frac{1}{\pi k_{\mathrm{F}} n_{\mathrm{t}}\left(E_{\mathrm{F}}\right)}\left(\frac{2 m E_{\mathrm{F}}}{\hbar^{2}}\right)^{1 / 2} \times \\
\times \sum_{l}\left(\frac{n_{l} n_{l+1}}{n_{l}^{(1)} n_{l+1}^{(1)}}\right)^{1 / 2} \sin \left(\delta_{l}-\delta_{l+1}\right) \\
\times \sum_{l^{\prime}}^{l}\left(2 l^{\prime}+1\right) P_{l^{\prime}}\left(1-\frac{q^{2}}{2 k_{\mathrm{F}}^{2}}\right)(2)
\end{aligned}
$$

where $k_{\mathrm{F}}$ is the free electron radius of the Fermi sphere and $P_{l}$ is the Legendre polynomial of order $l$. The rest of the symbols have been defined before in the calculation of $\eta$.

The sine term in the above expression and equivalently in the GG formula was obtained by evaluating the radial matrix element of the derivative of the RMTA potential [3]. Obviously the addition of the potential (1) into the RMTA potential will result in replacing the sine terms above by

$$
\sin \left(\delta_{l}-\delta_{l+1}\right)+\Delta V_{l, l+1} \text {. }
$$

Table I. - Calculated densities of states (total $n_{\mathrm{t}}$ and partial $n_{l}$ ) at the Fermi level and other basic quantities entering the formula for $T_{\mathrm{c}}$. Notation according to the text and references [1-4].

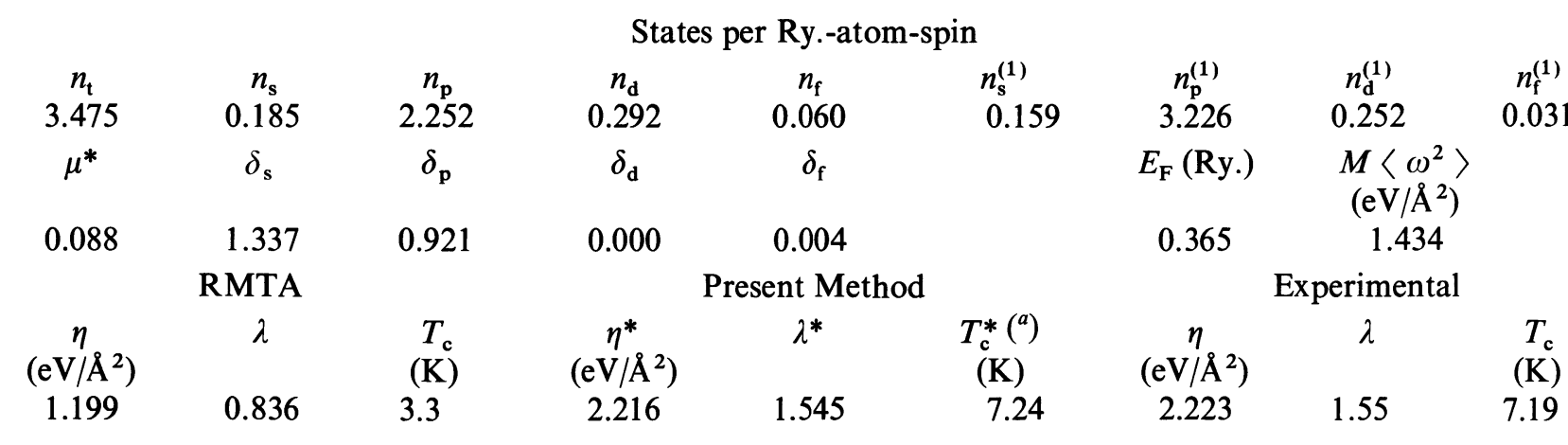


The terms $\Delta V_{l, l+1}$, being the radial matrix elements of the derivative of (1), are expressed in terms of $V_{0}$. The depth $V_{0}$ can be determined then by demanding that the new pseudopotential $\tilde{V}_{\text {ep }}(q)$ has the correct limit $[17,18]:-Z / 2 n_{\mathrm{t}}\left(E_{\mathrm{F}}\right)$ as $q$ approaches zero.

In figure 2 we have plotted both $\tilde{V}_{\text {RMTA }}(q)$ and $\tilde{V}_{\mathrm{ep}}(q)$ as a function of $q / 2 k_{\mathrm{F}}$. The deficiency of the RMTA can be seen from the fact that the RMTA pseudopotential reaches about half the correct value as $q$ approaches zero.

The new renormalized values of $n^{*}, \lambda^{*}$ and $T_{\mathrm{c}}^{*}$ are listed in table I. The agreement with the experimentally determined value of $\lambda$ is excellent.

Good agreement with experiment have been obtained before [11, 15, 19-22] using empirical pseudopotentials. In the present approach the pseudopotential was determined from our first principles band struc-

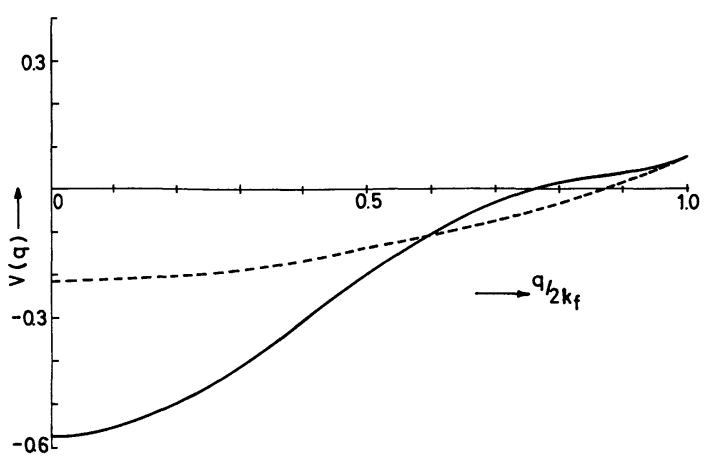

Fig. 2. - The RMTA (broken lines) and the corrected, $\tilde{V}_{\mathrm{ep}}(q)$, (solid lines) pseudopotentials for lead.

ture calculation and its limiting value as $q \rightarrow 0$, without any resort to experimental data.

\section{References}

[1] McMillan, W. L., Phys. Rev. 167 (1968) 331

[2] Gaspari, G. D. and Gyorffy, B. L., Phys. Rev. Lett. 29 (1972) 801

[3] Evans, R., Gaspari, G. D. and Gyorffy, B. L., J. Phys. F 3 (1973) 39.

[4] Allen, P. B. and Dynes, R. C., Phys. Rev. B 12 (1975) 905.

[5] Mattheiss, L. F., Phys. Rev. 151 (1966) 450.

[6] Koelling, D. D. and Harmon, B. N., J. Phys. C 4 (1971) 2064.

[7] Papaconstantopoulos, D. A., Zdetsis, A. D. and EconoMOU, E. N., Solid State Commun. 27 (1978) 1189.

[8] Mueller, F. M., Garland, J. W., Cohen, M. H. and BennemanN, K. H., Ann. Phys. 67 (1971) 19.

[9] Bennemann, K. H. and Garland, J. W., AIP Conf. Proc. 4 (1972) 103.

[10] Grimval, G., Phys. Scr. 14 (1976) 63 ; and references therein.

[11] Rietschel, H., Z. Phys. B 30 (1978) 271.
[12] JoHn, W., J. Phys. F. Metal Phys. 3 (1973) L 233.

[13] Rietschel, H., Z. Phys. B 22 (1975) 133.

[14] Winter, H. and RiEs, G., Z. Phys. B 24 (1976) 279.

[15] York, E. D. and Bardasis, A., Lett. Nuovo Cimento 2 (1971) 1228.

[16] Lee, M. J. G. and Heine, V., Phys. Rev. B 5 (1972) 3839.

[17] Ziman, J. M., Electrons and Phonons (Clarendon Press, Oxford) 1967.

[18] Heine, V., Nozieres, P. and Wilkins, J. W., Philos. Mag. 13 (1966) 741.

[19] Allen, P. B. and Cohen, M. L., Phys. Rev. 177 (1969) 704.

[20] Carbotte, J. P. and Dynes, R. C., Phys. Rev. 172 (1968) 476.

[21] Carbotte, J. P., Dynes, R. C. and Trofimenkoff, P. N., Can. J. Phys. 47 (1969) 1107.

[22] Allen, P. B. and Cohen, M. L., Phys. Rev. 187 (1969) 525 ; and references therein. 